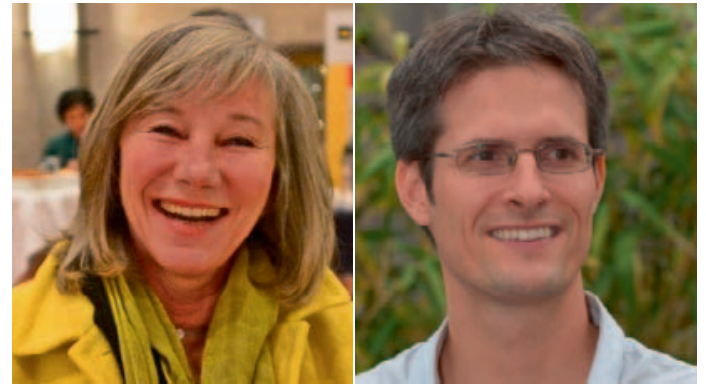

Dr. med. Anita

Simon Becker ${ }^{b}$

Meyer Hitz ${ }^{a}$

\section{Komplementäre und Integrative Medizin}

Die Komplementär- und Alternativmedizin (KAM) ist aus dem Gesundheitssystem der Schweiz nicht mehr wegzudenken. Gemäss Umfragen nutzt jeder zweite Schweizer bzw. jede zweite Schweizerin Methoden der KAM. Die Abstimmung "Ja zur Komplementärmedizin» vom 9. Mai 2009 hat mit einer überwältigenden Akzeptanz von 67\% gezeigt, dass KAM vom Volk gewünscht wird. Die Umsetzung der Kernforderungen der Initiative geht langsam, jedoch stetig vorwärts. Die KAM wird in die universitäre Ausbildung integriert; die nichtärztlichen Therapeutenberufe werden eidgenössisch geregelt.

Unter diesem Zeichen der positiven Akzeptanz fand Anfang Dezember 2014 in Solothurn bereits der 8. Kongress für Akupunktur und Chinesische Medizin statt. Dieser Kongress wird von der Assoziation Schweizer Aerztegesellschaften für Akupunktur und Chinesische Medizin (ASA) in Zusammenarbeit mit der Schweizerischen Berufsorganisation für Traditionelle Chinesische Medizin (SBOTCM) organisiert und durchgeführt.

Die Eröffnungsrede wurde von Frau Prof. Dr. med. Claudia Witt gehalten. Frau Prof. Witt ist eine international anerkannte Professorin und Forscherin in Komplementärer und Integrativer Medizin und lehrt seit Anfang 2014 an der Universität Zürich. Mit ihrem Lehrstuhl sowie dem Universitäts-Spital Zürich angegliederten Institut verbindet sie Schulmedizin mit der Komplementärmedizin - für

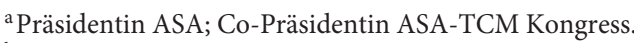

${ }^{\mathrm{b}}$ Co-Präsident ASA-TCM Kongress. die Akupunktur und die Chinesische Medizin in der Schweiz ein Glücksfall.

Anerkanntermassen gut steht es um die Evidenz der Akupunktur bei chronischen Schmerzen: Prof. Witt sowie Hugh MacPherson, ein renommierter Forscher im Bereich der Akupunktur aus England und ebenfalls Referent beim letzten TCM-Kongress, zeigten in ihren Metaanalysen mit Daten von über 18000 Patienten in insgesamt 29 Studien, dass die Akupunktur statistisch signifikant deutlich besser wirkt - sowohl als Placebo als auch als Schein-Akupunktur - und auch signifikant wirksamer ist als eine medizinische Standardtherapie [1]. Diese Resultate gelten für Schmerzzustände bei Migräne und Kopfschmerzen, bei muskuloskelettalen Schmerzen, bei Gonarthrose und bei Lumbalgie. Gerade bei diesen in der Praxis so häufigen Schmerzbildern ist Akupunktur einer Standardtherapie deutlich überlegen - allerdings ohne die gerade bei lang andauernder Anwendung von nichtsteroidalen Antirheumatika (NSAR) häufigen und oft auch gravierenden Nebenwirkungen!

Trotzdem bleiben viele Fragen ungeklärt: Welche Akupunktur wirkt am besten? Ist es besser, viele oder wenige Nadeln zu setzen? Wie viel Einfluss hat die Erfahrung des Behandlers? Untersuchungen zu diesen Fragen kommen zu unterschiedlichen und schwer interpretierbaren Resultaten. Einerseits, wie MacPherson in seiner Präsentation aufzeigte, weil diese vermutlich sehr geringen Unterschiede nur mit sehr grossen Fallzahlen erkannt werden könnten. Andererseits wies Witt darauf hin, dass Akupunktur als hochkomplexe Intervention eben nicht nur auf die spezifische Wirkung reduziert werden kann. Vielmehr ist die alltägliche Handlung eine Kombination von medizinisch-methodischen Kompetenzen mit kommunikativen und psychosozialen Skills. Die Erforschung der «soft factors» stellt ein nächstes Ziel dar. Für den Praxisalltag motivieren solche Einsichten. Sie liefern Inputs, Ideen und Erklärungen.

Weitere Anregungen bzw. Informationen zur Traditionellen Chinesischen Medizin und Akupunktur finden Sie auch in der vorliegenden Ausgabe der SCHWEIZERIsCHEN ZEITSCHRIFT FÜR GANZHEITSMEDIZIN - unter anderem im Interview mit Dr. Marko Nedeljković zum Thema Taiji, im Nachbericht zum oben erwähnten ASA TCM-Kongress oder auch im Beitrag «Entwicklung der Ohrakupunktur von der Antike bis heute» von Holzknecht und Wirz-Ridolfi.

\section{Literatur}

$\checkmark 1$ Vickers AJ, et al.: Acupuncture for chronic pain: individual patient data meta-analysis. Arch Intern Med 2012;172:1444-1453. 